\title{
Análise sobre o estilo de vida e comportamentos de risco dos universitários de Educação Física
}

\author{
Analysis of the lifestyle and risk behaviors of university Physical Education \\ Análisis del estilo de vida y comportamientos de riesgo de la Educación Física universitaria
}

Recebido: 29/03/2021 | Revisado: 09/04/2021 | Aceito: 13/04/2021 | Publicado: 24/04/2021

Thais Regina de Sousa

ORCID: https://orcid.org/0000-0002-3636-2420 Universidade Estadual do Paraná, Brasil

E-mail: thaisr72@hotmail.com

Sérgio Roberto Adriano Prati

ORCID: https://orcid.org/0000-0003-4375-2467 Universidade Estadual do Paraná, Brasil E-mail: srap@bol.com.br

\begin{abstract}
Resumo
Analisar o estilo de vida (EV) de universitários do curso de Educação Física (EF) dando ênfase aos aspectos entre sexo e entre nível de formação acadêmica. Participaram do estudo 114 estudantes de EF, da Universidade Estadual do Paraná, subdivididos em 54 do sexo masculino e 60 feminino, em 4 séries de formação acadêmica. Todos responderam ao questionário Estilo de Vida Fantástico (EVF) que compreende nove dimensões do estilo de vida individual. Observou-se que em média o EV dos universitários foi de $70( \pm 9,3)$ pts., não havendo diferença entre sexos, contudo, 56,1\% apresentaram EV dentro de um nível considerado adequado e saudável ( $\geq 70$ pts), e, o sexo masculino apresentou percentual maior $(59,2 \%)$ de estudantes com EV mais saudável em relação ao feminino $(53,3 \%)$. Identificou-se também que houve diferença estatística $(\mathrm{P}<0,05)$ entre o $\mathrm{EV}$ de estudantes ingressantes $\left(1^{\mathrm{a}}=72,8 \pm 9,3 \mathrm{pts}\right)$ em relação aos concluintes $\left(4^{\mathrm{a}}=66,2 \pm 7,8 \mathrm{pts}\right)$. Conclui-se que o EV dos universitários tem evoluído de forma negativa ao longo do curso de formação, sendo que de forma geral o sexo feminino apresenta condições relativas em condição pior que a do masculino.
\end{abstract}

Palavras-chave: Saúde do estudante; Comportamento de risco; Prevenção de doenças.

\begin{abstract}
Analyze the lifestyle of university students in the Physical Education course, emphasizing the aspects between sex and between academic education level. 114 Physical Education students from university in Paraná participated in the study, subdivided into 54 males and 60 females, in 4 academic series. All were answered to the Fantastic Lifestyle questionnaire, which comprises nine dimensions of individual lifestyle. It was observed that on average the lifestyle of the university students was $70( \pm 9.3)$ pts., With no difference between genders, however, $56.1 \%$ had an lifestyle within a level considered adequate and healthy $(\geq 70 \mathrm{pts})$, and, the male gender presented a higher percentage $(59.2 \%)$ of students with healthier lifestyle in relation to the female $(53.3 \%)$. He also identified that there was a statistical difference $(\mathrm{P}<0.05)$ between the lifestyle of freshmen $(1 \mathrm{st}=72.8 \pm 9.3 \mathrm{pts})$ in relation to the graduates $(4$ th $=66.2 \pm$ $7.8 \mathrm{pts})$. It is concluded that the lifestyle of the university students has evolved negatively throughout the course, and in general the female gender presents relative conditions in a worse condition than the male.
\end{abstract}

Keywords: Student health; Risk behavior; Disease prevention.

\section{Resumen}

Analizar el estilo de vida (EV) de los estudiantes universitarios en el curso de Educación Física (EF), enfatizando aspectos entre sexo y entre niveles de formación académica. En el estudio participaron 114 estudiantes de Educación Física de la Universidad Estatal de Paraná, subdivididos en 54 hombres y 60 mujeres, en 4 grados académicos. Todos fueron respondidos al cuestionario Fantastic, que comprende nueve dimensiones del estilo de vida individual. Se observó que en promedio el EV de los universitarios fue de 70 ( \pm 9.3$)$ pts., Sin diferencia entre géneros, sin embargo, el $56.1 \%$ tuvo un EV dentro de un nivel considerado adecuado y saludable ( $\geq 70 \mathrm{pts}$ ), y el masculino El género tuvo un porcentaje más alto $(59,2 \%)$ de estudiantes con EV más saludable en comparación con las mujeres $(53,3 \%)$. También se identificó que existía una diferencia estadística $(\mathrm{P}<0.05)$ entre el EV de los estudiantes entrantes $(1 \mathrm{er}=72.8 \pm$ 9.3 pts) en relación a los egresados (4to $=66.2 \pm 7.8$ pts). Conclui-se que o EV dos universitários tem evoluído de forma negativa ao longo do curso de formação, sendo que de forma geral o sexo feminino apresenta condições relativas em condição pior que a do masculino.

Palabras clave: Salud del estudiante; Comportamiento de riesgo; Prevención de Enfermedades. 


\section{Introdução}

O Estilo de Vida é um dos fatores essenciais para a manutenção da saúde física, mental e espiritual dos indivíduos, e quando esses níveis de saúde e bem-estar se encontram baixos podem afetar negativamente a vida das pessoas (NUNES, 2019). Sendo assim, o estudo sobre o estilo de vida das pessoas se torna fator preocupante, especialmente no âmbito acadêmico, período no qual requer atenção e adaptação por parte do estudante. Acredita-se que a passagem do ensino médio para o ensino superior faz como que o adolescente se depare com um novo conjunto de vivências e situações que implicam no seu estilo de vida, sendo isso muitas vezes comprometedor para toda sua vida futura.

Nahas (2006) enfatisa que as transformações sociais na vida das pessoas são decorrentes da percepção de bem-estar individual, porém, resultante de um conjunto de parâmetros também sócioambientais modificáveis ou não, que caracterizam condições em que vive o ser humano. Entre os socioambientais ele cita a moradia, o transporte, a segurança, a assistência médica, a educação, a cultura e o meio ambiente, já como parâmetros individuais destacam-se a hereditariedade (não modificável) e o estilo de vida. Como componente do estilo de vida podem ser destacados os hábitos alimentares, o controle do estresse, a atividade física habitual, o comportamento preventivo e os relacionamentos, ou seja, pode ser caracterizado por um conjunto de comportamentos adotados no dia-a-dia e representando um dos principais moduladores dos níveis de saúde e qualidade de vida (QV) das pessoas.

Schefer et al. (2015) relatam que para ter uma boa saúde com qualidade de vida é necessário possuir algumas características como ter paz de espírito, levar a vida com tranquilidade, ser feliz, ter saúde, gostar da vida, estar em equilíbrio, se sentir realizado, satisfeito e de bem consigo mesmo e com os outros. Para Linard et al. (2019) a aquisição de hábitos saudáveis se módula com a prática de atividade física, alimentação balanceada, comportamento preventivo e melhoria do sono, esses hábitos que podem reduzir os fatores de riscos para o desenvolvimento de doenças crônicas. Todas essas características compreendem aspectos do estilo de vida que podem ser modificáveis ao longo da vida, caso inadequados comprometimentos com o surgimento de doenças e disfunções pode ocorrer.

Luna et al. (2018) explicam que quando o estilo de vida é caracterizado com comportamentos negativos (comportamentos de risco à saúde - CRS) como o tabagismo, consumo excessivo de álcool e outros fatores como níveis insuficientes de atividade física, baixa tolerância ao estresse e relacionamentos afetivos ruins, hábitos alimentares inadequados e o uso de drogas ilícitas, esses hábitos, se, de forma crônica, levam jovens a se tornarem adultos com algum tipo de comprometimento da saúde. Nesse sentido, a adoção de hábitos saudáveis na vida dos jovens, poderia contribuir para a condição inversa, benefícios à saúde.

As mudanças no estilo de vida podem estar relacionadas às mudanças nas formas de se compreender o mundo, visto que a idade universitária culmina com o status de "maior idade". Neste sentido o período universitário demonstra ser um momento de grande importância no que diz respeito à saúde do indivíduo e desenvolvimento pessoal. Apesar de todos os benefícios sociais proporcionados pelo ingresso do jovem na universidade, a necessidade de inserção em um novo ambiente e inclusão a um grupo, sentimento de liberdade e independência, além do maior tempo gasto com atividades acadêmicas pode proporcionar um ambiente favorecedor de práticas não saudáveis. (Gasparotto, 2012; Souza; Baptista, 2017).

Nos estudos de Castaño et al. (2014) e Zeferino (2015) foi demonstrado que as populações universitárias eram altamente vulneráveis, sendo que as principais causas foram as influências associadas a mudança de vida, saída do ensino médio para o superior, na qual combinava com a disponibilidade de situações de consumo do álcool, drogas e o fumo, práticas comuns e consideradas livres para os jovens adultos no ambiente universitário. Autores ainda destacam que em decorrência dessa pseudo independência e autonomia em muitas ocasiões a falta de controle dos pais/responsáveis contribuíam para levar ao desenvolvimento de um estilo de vida negativo e de risco. Por outro lado, para Bomfim et al. (2017) e Cabral e Matos 
(2010), o ambiente universitário pode proporcionar aos jovens a possibilidade de constituir novos hábitos e comportamentos que serão decisivos para toda vida, além de uma nova rede de relações afetivas, e consequentemente favorecer o bem-estar psicológico e integração na faculdade. Isso decorreria caso ações fossem tomadas para contribuir para a formação profissional, bem como formação cidadã. Já quando a formação superior se relaciona com conteúdos que enfocam o estilo de vida ativo e saudável, como a formação em Educação Física, talvez essa condição pudesse ser mais favorável.

No caso de futuros profissionais da saúde, em especial aos de Educação Física, pode ser que o estudo da saúde, comportamentos preventivos e de risco, bem como, educação voltada para estímulo e conscientização de hábitos saudáveis de vida possam contribuir para melhoria da percepção pessoal sobre o estilo de vida saudável melhorando a própria vida do estudante (Silva et al. 2012; Belém et al., 2016). Alguns dos fatores decisivos para modificações destes comportamentos de riscos são: o conhecimento; a vontade e a atitude, sendo os fatores internos e as oportunidades, autoestima, fatores sócio culturais e fatores biológicos que se caracterizam por serem fatores externos ao indivíduo. Todos esses fatores estão relacionados com o estilo de vida de cada indivíduo (Nahas, 2006; Barbosa, 2012).

Enfim, mesmo, segundo Nahas (2006) relatando que seria muito simplicista acreditar que apenas a informação oriunda de uma formação acadêmica relacionada à saúde fosse determinante para mudança de comportamento do estudante, e, sendo essa uma questão complexa, como complexo é o comportamento humano em geral, as informações acadêmicas seriam relevantes para o grupo a que se destina, e, caso estiverem associadas a reais oportunidades para a adoção de novos hábitos talvez se transformasse em melhor condição de saúde geral. Assim o objetivo desse trabalho foi analisar o estilo de vida de estudantes de Educação física de uma universidade pública da cidade de Paranavaí-PR.

\section{Metodologia}

Esta pesquisa teve caráter descritivo e exploratório (Thomas; Nelson; Silverman, 2012) na qual coleta e análise informações de uma população específica sendo essas informações ainda pouco conhecidas. O estudo teve aprovação pelo Comitê de Ética da Universidade Estadual de Maringá CAAE 61069916.8.0000.0104 e parecer número 2.093.464.

Participaram do estudo 114 estudantes de Educação Física (EF) de uma universidade estadual do Paraná, subdivididos em 54 do sexo masculino (M) e 60 feminino (F). Todos responderam ao questionário Estilo de Vida Fantástico (EVF) (Anez; Reis; Petroski, 2008) composto por 25 questões de múltipla escolha em escala likert compreendendo nove dimensões do estilo de vida individual: Família (F); Atividade física (AF); Nutrição (N); Tabaco/Drogas (TD); Álcool (A); Segurança/Estresse (S); Tipo de comportamento (T); Introspecção (I); e Carreira (C). Valores de respostas variam de 0 a 4 pontos, totalizando o máximo de 100 pontos, sendo que quanto maior a pontuação melhor, mais saudável e seguro é o estilo de vida.

A aplicação dos questionários foi realizada em sala de aula com todos alunos presentes e que aceitaram participar da pesquisa. O instrumento questionário de EV Fantástico é autoexplicativo, porém os pesquisadores estavam em sala para orientarem inicialmente no preenchimento do formulário. Para análise estratificada foi verificado resultado proporcional em cada domínio, por sexo e por nível de ensino.

Para uma análise dicotomizada dos resultados foram consideradas as respostas com valores de 0,1 e 2 como “inadequadas" (EV menos saudável e seguro) e respostas 3 e 4 como "adequadas” (EV mais saudável e seguro).

Os dados foram analisados através da estatística descritiva com valores expressos em percentual, média e desvio padrão geral, por domínio do estilo de vida, por série e por sexo. Os testes "t" de Student e análise de variância (ANOVA, post hoc Scheffé test) foram utilizados para comparação entre grupos. Em todos os testes estatísticos o grau de confiança foi de $95 \%$. 
Quadro 1 - Características dos acadêmicos de Educação Física que compuseram o estudo.

\begin{tabular}{|lcc|}
\hline \multicolumn{1}{|c|}{ Características } & $\mathbf{N}$ & $\%$ \\
\hline Sexo & 61 & 53,5 \\
Feminino & 53 & 46,5 \\
Masculino & 114 & 100 \\
Total & & 14,9 \\
& & 18,4 \\
Ingressantes & 17 & 33,3 \\
Feminino & 21 & 12,3 \\
Masculino & 38 & 7 \\
Total & & 19,3 \\
& & 2,3 \\
Concluintes & 14 & 21,9 \\
Feminino & 8 & 47,3 \\
Masculino & 22 & \\
Total & & \\
Series intermediarias & 29 & \\
Feminino & 25 & \\
Masculino & 54 & \\
Total & & \\
\hline N= número de acadêmicos & porcentagem & \\
\hline
\end{tabular}

Fonte: Autores.

\section{Resultados}

Os resultados serão apresentados em princípio analisando o estilo de vida geral entre todos os estudantes comparandoos entre sexos, a seguir serão apresentados os resultados das comparações entre níveis de ensino e por sexos, posteriormente a análise será mostrada a partir das dimensões do estilo de vida observando de forma transversal os ingressantes e concluintes.

Tabela 1. Estilo de vida de estudantes de Educação Física. Análise geral e por sexo. Valores expressos em média, desvio padrão e percentual de casos adequados e não adequados. Significativo quando $\mathrm{P}<0,05^{*}$ :

\begin{tabular}{cccccccccc}
\hline PONTOS & \multicolumn{2}{c}{ TODOS } & \multicolumn{3}{c}{ MASCULINO } & \multicolumn{3}{c}{ FEMININO } \\
\hline & $\mathbf{n}(\%)$ & Média & S & $\mathbf{n}(\%)$ & Média & S & n (\%) & Média & S \\
\hline Todos & $114(100)$ & 70,0 & 9.3 & $54(47,3)$ & 71,0 & 8,7 & $60(52,6)$ & 69,2 & 9,7 \\
Adequados & $64(56.1)$ & 76,8 & 4.6 & $32(59,2)$ & 76,9 & 4,9 & $32(53,3)$ & 76,7 & 4,4 \\
Inadequados & $50(43.8)$ & 61,4 & 6.0 & $22(40,7)$ & 62,4 & 5,2 & $28(46,6)$ & 60,6 & 6,5 \\
\hline
\end{tabular}

Nota* Adequados quando o resultado for $\geq 70$ pts.; $S=$ desvio padrão; $N=$ número. Fonte: Autores.

A Tabela 1 identifica que não há diferença significante $(P>0,05)$ entre o estilo de vida de estudantes de Educação física entre os sexos entre masculino (71,0 $0 \pm 8,7 \mathrm{pts})$ e feminino $(69,2 \pm 9,7 \mathrm{pts})$, contudo observa-se que o sexo masculino apresenta uma prevalência maior $(59,2 \%)$ de estudantes com estilo de vida adequado em relação ao feminino (53,3\%), porém não significantes. 
Tabela 2. Estilo de vida de estudantes de Educação Física. Comparação entre os níveis de ensino do sexo masculino

\begin{tabular}{cccccc}
\hline \multicolumn{2}{c}{ MASCULINO } & & & Adequados & Inadequados \\
\hline Nível de ensino & $\mathbf{n}(\boldsymbol{\%})$ & Média & $\mathbf{S}$ & $\mathbf{n}(\boldsymbol{\%})$ & $\mathbf{n}(\boldsymbol{\%})$ \\
\hline $1^{\mathbf{o}}$ & $21(38,9)$ & $* \mathbf{7 4 , 8}$ & 7,7 & $16(29,6)$ & $5(9,3)$ \\
$2^{\text {o }}$ & $15(27,8)$ & 69,2 & 8,7 & $8(14,8)$ & $7(13)$ \\
$3^{\text {o }}$ & $10(18,5)$ & 69,9 & 10,2 & $7(12,9)$ & $3(5,5)$ \\
$4^{\text {o }}$ & $8(14,8)$ & $* \mathbf{6 5 , 8}$ & 5,3 & $1(1,9)$ & $7(12,9)$ \\
Total & $54(100)$ & 71,0 & 8,7 & $32(59,2)$ & $22(40,7)$ \\
\hline
\end{tabular}

* Adequados quando o resultado for $\geq 70$ pts; $\mathrm{S}=$ Desvio Padrão; N= número; Significativo quando $\mathrm{P} \leq 0,05$. Fonte: Autores.

Tabela 3. Estilo de vida de estudantes de Educação Física. Comparação entre os níveis de ensino do sexo feminino.

\begin{tabular}{|c|c|c|c|c|c|}
\hline \multicolumn{2}{|c|}{ FEMININO } & \multirow[b]{2}{*}{ Média } & & \multirow{2}{*}{$\begin{array}{c}\text { Adequados } \\
\text { n }(\%)\end{array}$} & \multirow{2}{*}{$\begin{array}{c}\text { Inadequados } \\
\mathbf{n}(\%)\end{array}$} \\
\hline Nível de ensino & n (\%) & & $\mathbf{S}$ & & \\
\hline $1^{\circ}$ & $17(28,3)$ & $* 70,4$ & 10,7 & $11(18,3)$ & $6(10)$ \\
\hline $2^{o}$ & $11(18,3)$ & 69,0 & 12,8 & $5(8,3)$ & $6(10)$ \\
\hline $3^{\circ}$ & $18(30)$ & 70,3 & 7,2 & $11(18,3)$ & $7(11,7)$ \\
\hline $4^{\circ}$ & $14(23,3)$ & $* 66,5$ & 9,2 & $5(8,3)$ & $9(15)$ \\
\hline Total & $60(100)$ & 69,2 & 9,7 & $32(53,3)$ & $28(46,7)$ \\
\hline
\end{tabular}

* Adequados quando o resultado for $\geq 70$ pts; Pts= Pontos; $\mathrm{S}=$ Desvio Padrão; N= número; Significativo quando P $\leq 0,05$. Fonte: Autores.

Nas Tabelas 2 e 3 pode-se observar que em ambos os sexos houve diferença significativa entre as médias do EV de estudantes ingressantes em relação aos concluintes. Essa condição é mais evidente no sexo masculino (ingressantes = 74,8pts; concluintes $=65,8 \mathrm{pts})$. Assim, demonstra-se que mesmo após anos de estudo em temáticas relacionadas a área da saúde e comportamento saudável os universitários concluintes apresentam em média EV mais inadequado do que os colegas de séries iniciais. Pode-se observar que no sexo masculino à medida que os quatro anos de curso passam a prevalência de estudantes com EV adequado cai gradativamente $(29,6 \% ; 14,8 \% ; 12,9 \% ; 1,8 \%)$. No sexo feminino essa condição é um pouco diferente, pois há nesse caso a prevalência de casos adequados de forma parecida das ingressantes com estudantes de $3^{\mathrm{a}}$. Série (18,3\%), porém, entre ingressantes e concluintes a diferença entre a prevalência de moças com EV adequado também se destaca negativamente $\left(1^{\mathrm{a}}=18,3 \% ; 4^{\mathrm{a}} 8,3 \%\right)$ 
Tabela 4. Estilo de vida de estudantes universitários de Educação Física ingressantes por dimensão de EV. Valores em média de pontos na dimensão, desvio padrão e percentual de casos inadequados.

\begin{tabular}{|c|c|c|c|c|c|}
\hline \multicolumn{6}{|c|}{ INGRESSANTES } \\
\hline Dimensão do EV & $\mathrm{N}$ & Média & $\mathrm{S}$ & Inadequado (pts.) & \%Inadequado \\
\hline $\mathrm{F}$ & 38 & 6,6 & 1,3 & $<6$ & $6(15,7)$ \\
\hline A & 37 & 5,1 & 1,9 & $<6$ & $19(51,3)$ \\
\hline $\mathrm{N}$ & 36 & 6,6 & 2,5 & $<9$ & $28(77,7)$ \\
\hline $\mathrm{T}$ & 33 & 14,3 & 1,7 & $<13$ & $4(12,1)$ \\
\hline A & 32 & 11,0 & 1,4 & $<10$ & $4(12,5)$ \\
\hline S & 37 & 13,7 & 3,2 & $<15$ & $24(74,8)$ \\
\hline $\mathrm{T}$ & 38 & 5,0 & 1,8 & $<6$ & $23(60,5)$ \\
\hline I & 38 & 8,3 & 2,0 & $<9$ & $20(52,6)$ \\
\hline $\mathrm{C}$ & 38 & 3,1 & 0,9 & $<3$ & $8(21,0)$ \\
\hline
\end{tabular}

EV= Estilo de vida; Família (F); Atividade física (A-F); Nutrição (N); Tabaco/Drogas (T-D); Álcool (A); Segurança/Estresse (S); Tipo de comportamento (T); Introspecção (I); e Carreira (C).; Pts= Pontos; $\mathrm{S}=$ Desvio Padrão; $\mathrm{N}=$ número. Fonte: Autores.

Tabela 5. Estilo de vida de estudantes universitários de Educação Física concluintes por dimensão. Valores em média, desvio padrão e percentual de casos inadequados:

\section{CONCLUINTES}

\begin{tabular}{cccccc}
\hline Dimensão do EV & N & Média & S & Inadequado (pts.) & \% inadequados \\
F & 22 & 6,8 & 1,1 & $<6$ & $4(18,1)$ \\
A & 21 & 4,3 & 2,2 & $<6$ & $14(66,6)$ \\
N & 20 & 6,3 & 2,3 & $<9$ & $17(85,0)$ \\
T & 20 & 13,9 & 2,4 & $<13$ & $3(15,0)$ \\
A & 20 & 8,1 & 3,6 & $<10$ & $10(5,0)$ \\
S & 21 & 12,7 & 2,5 & $<15$ & $17(77,2)$ \\
T & 22 & 3,7 & 1,0 & $<6$ & $21(95,4)$ \\
I & 22 & 7,7 & 2,2 & $<9$ & $13(59,0)$ \\
C & 22 & 3,0 & 0,6 & $<3$ & $5(22,7)$ \\
\hline
\end{tabular}

EV= Estilo de vida; Família (F); Atividade física (A-F); Nutrição (N); Tabaco/Drogas (T-D); Álcool (A); Segurança/Estresse (S); Tipo de comportamento (T); Introspecção (I); e Carreira (C).; Pts= Pontos; $\mathrm{S}=$ Desvio Padrão; N= número. Fonte: Autores.

$\mathrm{Na}$ análise do estilo de vida dos estudantes de Educação física por dimensão, segundo os critérios do instrumento utilizado, percebeu-se que em média ingressantes e concluintes apresentaram resultados menores do que o adequado para AF, N, S, T e I. Os concluintes ainda acrescentaram a dimensão AL com inadequado. Ou seja, se há diferença entre ingressantes e concluintes, de uma forma geral, parece que fator consumo de álcool $\left(1^{\circ}=11 \mathrm{pts} ; 4^{\mathrm{a}} .=8,1 \mathrm{pts}\right)$ dos universitários de maior nível acadêmico é que contribui para que essa característica torne diferente a média entre os diferentes níveis. Por fim, somente na dimensão família e amigos (FA) é que os valores em média dos concluintes (FA=6,8pts) é ligeiramente superior ao dos ingressantes $(6,6 \mathrm{pts})$, todavia não é significativa. 
Em relação a prevalência de casos alterados por dimensão na comparação transversal entre ingressantes e concluintes, em todas as dimensões os universitários em fase de conclusão de curso apresentam mais casos alterados do estilo de vida em relação aos ingressantes. Destacam-se as dimensões atividade física $(\mathrm{AF}=66,6 \%)$, nutrição $(\mathrm{N}=85 \%)$, álcool $(\mathrm{AL}=50 \%)$ e tipo de comportamento $(\mathrm{T}=95,4 \%)$ alterados.

Mesmo considerando que o processo de desenvolvimento acadêmico não tem contribuído para a melhoria do EV dos universitários, cabe ressaltar que dentre os ingressantes participantes dessa pesquisa observou-se que 51,3\%, 77,7\%, 74,8\%, $60,5 \%$ e $52,6 \%$ respectivamente dos ingressantes estavam inadequados em AF, N, S, T e I, o que prevê ao longo dos anos subsequentes potencial piora dessas condições caso não haja alguma tendência de alteração.

\section{Discussão}

Como síntese final dos resultados expressos pelo estudo, na comparação entre os sexos feminino e masculino de ambos níveis de ensino não há diferença estatisticamente significativa. Pode-se julgar diante os resultados obtidos que os ingressantes em geral, apresentam melhores índices de um estilo de vida adequado comparado aos concluintes, essa condição é mais evidente no sexo masculino, e os concluintes por terem um embasamento teórico relevante sobre a promoção da saúde e de estilos de vida saudáveis deveriam apresentam outro tipo de comportamento em relação aos ingressantes.

$\mathrm{Na}$ análise do estilo de vida por dimensão os ingressantes e concluintes apresentaram resultados menores do que o adequado para $\mathrm{AF}, \mathrm{N}, \mathrm{S}$, T e I, e ainda os concluintes acrescentaram $\mathrm{AL}$ com inadequados, com isso o estudo mostrou que a prevalência dos maus hábitos aumenta juntamente com o nível de ensino dos acadêmicos.

Nos escores gerais dos nove domínios que compõem o questionário FANTÁSTICO (Añez et al., 2008), realizou-se uma análise de cada um dos domínios para testar as possíveis modificações no estilo de vida. Como as respostas às questões seguem uma escala que representam categorias de resposta, além de que os escores dos domínios não apresentam distribuição normal. Os resultados encontrados no ambiente acadêmico têm influência de inúmeras mudanças na vida dos universitários, como modificações emocionais, nutricionais, ansiedade e o de maior relevância as novas amizades, portanto podem intervir no comportamento pessoal e alimentar inadequado (Vieira et al., 2002).

Os resultados semelham aos dados referenciados na literatura de (Bomfim, 2017; Mello, 2016; Sousa et al., 2016; Oliveira, 2017; Barbosa, 2015) Sendo que para os resultados desses trabalhos foi utilizado o mesmo questionário do presente estudo, que identificam fatores de ordem social, cultural, socioeconómica além de outros como estando implicados nos resultados das mais distintas variáveis de saúde, como avaliando o estilo de vida ou o próprio estado de saúde dos universitários, alguns dos artigos ainda apresentou algumas associações entre doenças ligadas ao estilo de vida que podem ser desencadeadas durante a graduação se o acadêmico não tomar os devidos cuidados, existem muitos métodos preventivos que podem evitar muitos quadros de doenças físicas e também psicológicas que os acadêmicos devem ter a devida consciência.

Os resultados obtidos neste estudo não condizem com os esperados, pois por se tratarem de acadêmicos da área de formação ligada a saúde e os totais das pontuações das variáveis não terem atingidos o valor limítrofe (>70 pts.) é algo para ser investigado mais a fundo. Esta evidencia pode passar ao entendimento de que os conhecimentos adquiridos pelos alunos não estão sendo postos em pratica em suas próprias vidas, falta a conscientização e responsabilidade por parte desses acadêmicos. Este papel se faz parte da essência do curso, pois futuramente esses futuros profissionais irão avaliar o estado de saúde de outras pessoas, por tal conhecimento e a mudança nas atitudes se torna fundamental, o papel deverá ser atribuído à universidade enquanto instituição, também ela, promotora da saúde. Desta forma a educação a saúde deverá ocupar um papel determinante na formação desses acadêmicos, que cabe sem dúvidas ao sistema educativo dependentes pelo modo de construir respostas preventivas que irão fazer parte da concepção global da pessoa como cidadão. 
Os resultados do presente estudo podem ser utilizados por professores, familiares, e profissionais da saúde que configuram um papel importe na vida dos acadêmicos e são determinantes na mudança e prevenção de risco maiores na vida desta população jovem. Com isso à necessidade das instituições desenvolverem programas para facilitar, promover e passar o conhecimento para os acadêmicos sobre as conduta e escolhas pessoais ligadas com os comportamentos de saúde, para facilitar a adoção de novos hábitos de um estilo de vida mais saudável e para que assim o acadêmico transfira esses conhecimentos para sua vida social.

\section{Conclusão e Considerações Finais}

O EV dos estudantes de Educação física evolui negativamente ao longo dos anos de curso, na qual se observou que no sexo masculino essa condição é mais presente. De outro modo, percebeu-se que de modo específico, quando analisadas as dimensões do EV mais relacionadas aos conteúdos abordados ao longo do curso como atividade física, nutrição, estresse, combate ao uso de álcool, tabaco e drogas e tipo de comportamento, em ambos os sexos há maior prevalência de casos alterados em estudantes em fase de conclusão de curso. Todavia, nos ingressantes as perspectivas também não são boas frente as mesmas dimensões.

O presente estudo com a apresentação de dados coletados, mostra que a universidade por ser um período de transição entre a fase juvenil e adulta, gera transformações evidentes na vida dos indivíduos, e a universidade tem o papel de facilitar essa transição, promovendo a criação de contextos que visem à integração total do indivíduo para a permanência com qualidade de ensino dos estudantes até a conclusão do curso. Este estudo ainda evidência que há necessidade do conhecimento sobre saúde e estilo de vida saudável na vida desses estudantes, e que a intervenção se faz necessária para contribuir na melhora do EV nas diferentes dimensões do estilo de vida.

Como apresentou a pesquisa, o ingresso no ensino superior acarreta em inúmeras transformações e com elas aparecem as vulnerabilidades e os comportamentos de riscos dos universitários, nesse panorama sugere-se a necessidade de futuras pesquisas que apresentam as instabilidades psicológicas e comportamentais ocorridas no ambiente acadêmico e suas influências sobre o estilo de vida e desempenho acadêmico dos universitários na graduação, objetivando contribuir para mudanças desses comportamento, sendo necessário programas de promoção da saúde inseridos nas próprias instituições de ensino.

\section{Agradecimentos}

Agradeço ao grupo de pesquisa PAFiDH no qual desenvolvi a pesquisa e a Fundação Araucária por financiar este projeto.

\section{Referências}

Añez, C. R. R., Reis, R. S. \& Petroski, E. L. (2008). Versão Brasileira do Questionário "Estilo de Vida Fantástico": Tradução e Validação para Adultos Jovens. Arquivo Brasileiro de Cardiologia. 91(2), 102-9

Barbosa, R. M. dos S. P. (2012). Resenha do livro "Atividade física, saúde e qualidade de vida: Conceitos e sugestões para um estilo de vida ativo", de Markus Vinicius Nahas. Rev. Bras. Ciênc. Esporte, 34(2), 513-518

Barbosa, R. R., Martins M. C. G., Carmo, F. P. T., Jacques, T. M., Serpa, R. G., Calil, O. A.\& Barbosa, L. F. M. (2015). Estudo sobre estilos de vida e níveis de estresse em estudantes de medicina. Int J Cardiovasc Sci, 28(4), 313-9.

Belem, I. C., Rigoni, P. A. G., Santos, V. A. P., Vieira, J. L. L. \& Vieira, L. F. (2016). Associação entre comportamentos de risco para a saúde e fatores sociodemográficos em universitários de educação física. Motricidade, 12(1), 3-16.

Bomfim, R. A., Mafra, M. A. S., Gharib, I. M. S., De-Carli, A. D. \& Zafalon, E. J. (2017). Fatores associados ao estilo de vida fantástico de universitários brasileiros-uma análise multinível. Revista CEFAC, 19(5), 601-610. 
Research, Society and Development, v. 10, n. 4, e58610414432, 2021

(CC BY 4.0) | ISSN 2525-3409 | DOI: http://dx.doi.org/10.33448/rsd-v10i4.14432

Cabral, J. \& Matos, P. (2010). Preditores da adaptação à Universidade: o papel da vinculação, desenvolvimento psicossocial e coping. Psychologica Imprensa da Universidade de Coimbra. 1, 55-77.

Castaño-perez, G. A. \& Calderon-vallejo, G. A. (2014). Problemas associados ao consumo de álcool em estudantes universitários. Revista Latino-Americana de Enfermagem, 22(5), 739-746.

Sousa, K. J. Q. \& Borges, G. F. (2016). Estilo de vida, atividade física e coeficiente acadêmico de universitários do interior do amazonas-brasil.

Souza, M. S. \& Baptista, M. N. (2017). Associações entre suporte familiar e saúde mental. Psicologia Argumento, 26(54), $207-215$.

Gasparotto, G. S. (2012). Associação entre o nível de atividade física e fatores de risco cardiovascular em universitários da UFPR.

Linard, J. G., Mattos, S. M., Almeida, I. L. S., Silva, C. B. A. S. \& Moreira, T. M. M. (2019). Associação entre estilo de vida e percepção de saúde em estudantes universitários. Journal of Health \& Biological Sciences, 7(4), 374-381.

Luna, A. A., Molari, M., Primo, S. H., Bispo, N. N. C., Ossada, V. A. Y., \& Costa, V. S. P. (2018). Caracterização do estilo de vida de universitários do ensino superior a distância. Journal of Health Sciences, 20(1), 40-44.

Mello, S. H. (2016). Perfil do estilo de vida e nível de atividade física de estudantes universitários de educação física. Trabalho de Conclusão de Curso. Universidade Tecnológica Federal do Paraná.

Nahas, M. V. (2006). Atividade física, saúde e qualidade de vida - conceitos e sugestões para um estilo de vida ativo. Midiograf. (3a ed.).

Nunes, M. A. P. D., Costa, G. L., Viana, F. C., Ferreira, C. M., Pereira, R. C. B \& Nascimento, L. C. G. (2019). Estilo de vida de universitários. Psicologia e Saúde em debate, 5(2), 102-102.

Oliveira, T. L. Pôrto, E. F., Künple, C. Campelo, Pucci, S. C., Leite, J. R. O. \& Almeida, S. C. (2017). Associação entre jornadas de trabalho e estilo de vida. Life Style, 4(2), 55-71.

Scheffer,M. L. C.; Pilatti, L. A.; Kovaleski, J. L. (2015). Qualidade Vida e Atividade Física na Literatura. Revista Espacios: $36(3)$, 7.

Silva, D. A. S., Pereira, I. M. M., Almeida, M. B., Silva, R. J. S. \& Oliveira, A. C. C. (2012). Estilo de Vida de Acadêmicos de Educação Física de uma Universidade Pública do Estado do Sergipe, Brasil. Revista Brasileira de Ciência do Esporte, 34(1), 53-67.

Thomas, J., Nelson, J. \& Silverman, S. J. (2012). Métodos de pesquisa em atividade física e saúde. (3a ed.), Artmed Editora.

Vieira, V. C. R., Priore, S. E., Ribeiro, S. M. R., Franceschini, S. C. C. \& Almeida, L. P. (2002). Perfil socioeconômico, nutricional e de saúde de adolescentes recém-ingressos em uma Universidade pública brasileira. Revista de Nutrição, 15(3), 273-282.

Zeferino, M. T., Hamilton, H., Brands, B., Wright, M. G. M. \& Cumsille, A. K. (2015). Consumo de drogas entre estudantes universitários: família, espiritualidade e entretenimento moderando a influência dos pares. Texto \& Contexto-Enfermagem, 24, 125-135. 